\title{
Electrocerebral Recovery During the Intracarotid Amobarbital Procedure: Influence of Interval Between Injections
}

\author{
Linda M. Selwa, ${ }^{*} \dagger$ Henry A. Buchtel, and $\ddagger$ Thomas R. Henry \\ Department of Neurology, University of Michigan, *Ann Arbor VA Medical Center and †Department of Psychiatry and \\ Psychology, University of Michigan; and $\ddagger$ Department of Neurology, Emory University, Ann Arbor, Michigan, U.S.A.
}

\begin{abstract}
Summary: Purpose and Methods: During the intracarotid amobarbital procedure (IAP) at the University of Michigan, continuous scalp EEG monitoring guides the timing for presentation of memory items and postinjection testing. Most of our patients have undergone bilateral injections. The interval between injections varied from 22 to $60 \mathrm{~min}$, depending on the test and recovery time, as well as the time to catheterize the second side. After noting a trend toward prolonged electrographic recovery following the second injection, we tested our clinical impression that recovery of the second hemisphere may be influenced by (a) the time between injections and (b) which hemisphere is injected first (epileptogenic or nonepileptogenic).
\end{abstract}

To study these questions, we analyzed EEG recovery data from 48 consecutive IAPs. Approximately half the patients had the epileptogenic side injected first.

Results: We found that (a) electrographic recovery after the second injection is prolonged if the interval between bilateral injections is less than 40 minutes and (b) electrographic recovery is more rapid after injection of the epileptogenic hemisphere.

Conclusions: We now recommend waiting at least $45 \mathrm{~min}$ between injections. The pathophysiology of more prolonged amobarbital effect on the nonepileptogenic hemisphere than on the epileptogenic hemisphere remains unclear. Key Words: Amobarbital-Intracarotid-Epilepsy-Temporal lobeMemory-Epilepsy partial-Injections-Intraarterial.
For more than a decade, Wada's intracarotid amobarbital test was used exclusively to localize language function (1). In 1962, Milner (2) proposed that the procedure be used to identify patients at risk for anterograde global amnesia after anterior temporal lobectomy (ATL). While the role of the intracarotid amobarbital procedure (IAP) in localizing language function has remained largely unchanged, significant controversy has arisen about the value of the IAP in (a) noninvasive lateralization of the epileptogenic zone (3-7) and (b) prediction of postoperative memory function $(6,8,9)$. The role of the IAP in predicting both seizure outcome and postoperative cognition depends on consistent, accurate, bilateral memory testing during the procedure. To facilitate investigation of these issues, a standardized IAP protocol, with equivalent injections of the two hemispheres, must be developed.

At our institution, concurrent monitoring of EEG activities during the IAP has become an important tool for interpreting changes in the patient's level of alertness, excluding the possibility of ictal activity, and determin-

\footnotetext{
Accepted May 29, 1997.

Address correspondence and reprint requests to Dr. L. M. Selwa at University of Michigan, Department of Neurology, 1914 Taubman Center/0316, 1500 E. Medical Center Drive, Ann Arbor, Michigan 48109, U.S.A.
}

ing the extent of amobarbital effect in the hemisphere contralateral to injection. Previous studies have demonstrated an excellent correlation between the type of online visual analysis we perform and automated quantitative techniques (10). Data available during the IAP provide the most useful information for optimizing the timing of injections and behavioral testing. Qualitative EEG has some additional advantages while the patient is being tested: (a) it allows the electroencephalographer to evaluate different types of artifact effectively (including myogenic, kinesogenic and oculomotor), (b) it allows the electroencephalographer some degree of ability to distinguish between amobarbital-induced slowing and generalized slowing at drowsiness, and (c) it provides very rapid indications of any of the patient's usual ictal events.

Recently, we undertook an investigation of the optimal sequence and timing of bilateral IAPs. Traditionally, some centers have chosen to perform hemispheric injections on separate days-apparently preferring to avoid potential interaction between serial injections, but risking an increased complication rate with separate catherization procedures. The overall morbidity data reported by Rausch (11) shows that $79 \%$ of centers have encountered a $0-1 \%$ risk, while $21 \%$ of centers reported a $1-5 \%$ incidence of morbidity. According to a survey of IAP 
centers in 1992 (12), 60\% of bilateral IAPs are performed on the same day, injecting the epileptogenic hemisphere first. A brief review of protocols for $10 \mathrm{ma}$ jor centers which recently published IAP data shows that 3 perform the injections on subsequent days, 3 allow $45-60 \mathrm{~min}$ before the second injection, and 4 wait $\leqslant 30$ min between injections.

At our center, preliminary investigation yielded an indication that (a) the electrographic recovery time after the nonepileptogenic hemisphere is injected may be longer than recovery after injection of the epileptogenic hemisphere and (b) the length of the interval between first and second injections might influence the rate of electrographic recovery after the second test. We were interested in investigating the optimal interval between the 2 injections - that is, to determine the waiting period that would eliminate the residual effect of the amobarbital on electrographic changes and which might possibly, avoid behavioral dysfunction.

\section{METHODS}

\section{Subjects}

A total of 48 patients had IAPs with complete EEGCCTV monitoring at the University of Michigan between April 1990 and April 1993. All were evaluated as part of an epilepsy surgery protocol for treatment of refractory partial epilepsy (13). The group, (Table 1), includes 26 males and 22 females. Epileptogenic zones were subsequently resected in all patients; there were 23 right- and 25 left-sided resections. All of our patients had left-hemisphere language dominance or predominance, except for 1 right-handed woman, who had righthemispheric language predominance. Pathologically, 10 patients were found to have progressive or nonprogressive lesions, including 4 gliomas, 1 ganglioglioma, 2 epidermoid cysts, 1 hamartoma, and 2 vascular malformations. Six of the patients had only unilateral IAPs; 42 bilateral tests were available for review.

To minimize the risk of unexpected cognitive changes during localization procedures, we perform IAPs only after definitive identification of ictal onset zones, i.e., after any intracranial interventions that might alter function of nonresected tissue. In 22 of the bilateral procedures performed, the epileptogenic hemisphere was in-

TABLE 1. IAP demographics

\begin{tabular}{lll}
\hline Sex & 26 male & 22 female \\
Laterality of resection & 25 left & 23 right \\
Languagc predominance & 47 left & 1 right \\
Pathology & 38 mesial TLE & 10 lesional \\
$\begin{array}{l}\text { Side of first injection } \\
\quad \text { (bilateral) }\end{array}$ & 22 epileptiform & 20 nonepileptiform \\
Unilateral/bilateral IAP & 6 unilateral & 42 bilateral \\
\hline
\end{tabular}

IAP, intracarotid amobarbital procedure. jected first, and in 20 others, the nonepileptogenic side was injected first. In cases with any bilateral ictal onsets, IAP data were reviewed after the procedure to evaluate the correspondence of ictal sampling and IAP testing in identifying the more dysfunctional hemisphere.

\section{IAP}

The IAP followed a standard protocol, a modification of the method pioneered at the Montreal Neurologic Institute (14). Following transfemoral catheterization, machine-injected intracarotid angiography was carried out in each hemisphere immediately before manual injection of $125 \mathrm{mg}$ of amobarbital in $5 \mathrm{cc}$ sterile water over $3 \mathrm{~s}$. Continuous EEG-CCTV monitoring began with a baseline 16-channel tracing for approximately $25 \mathrm{~min}$ before injection. The electrographic data were carefully monitored by a qualified electroencephalographer (TRH or LMS). At the time of the injection, the patient was asked to count, to grip both of the examiner's hands continuously and to wiggle toes bilaterally. Behavioral assessment began with preinjection testing by a qualified neuropsychologist (HAB) and continued throughout the procedure.

When electrographic slowing over the injected hemisphere had dissipated from primarily delta to primarily theta frequencies (usually 2-3 min), 5 memory items were presented (rarely, the presentation had to be delayed because the patient did not yet seem attentive). The test stimuli consisted of (a) an object viewed and palpated in the hand ipsilateral to the injection, (b) 2 simple line drawings, (c) a sentence spoken by the examiner and repeated by the patient if possible, and (d), a large word printed on a card. No items were presented after strength had recovered to three-fifths in the hand contralateral to injection. Periodic evaluation of motor and sensory function and visual fields was conducted; motor function was assessed by a neurologist and recorded on the tracing by a technologist. Motor function was judged normal when grip strength and finger movements had returned to baseline. Assessment of both spontaneous and recognition memory was conducted after full motor recovery and after the return of a 10-s epoch of the patient's usual baseline EEG frequencies. A "passing" grade was considered to be convincing recall of 3 or more of the 5 items. After the procedure, patients were questioned about any physical or cognitive changes noted.

\section{Analysis and statistics}

EEG paper tracings were reviewed retrospectively, blinded to patient identity or side of epileptogenesis. The mean and standard deviation of 6 electrographic and motor variables were ascertained for each IAP. These variables are: $(a, b)$, time to return to baseline EEG for first and second injections; (c,d), time to return to baseline 
motor abilities for first and second injections; and (e,f), time to delta/theta transition for first and second injections. Paired t-tests and Pearson correlation coefficients were used to investigate the possible effects of 3 factors on the time required for electrographic activity to return to baseline. Factors investigated included effects of firstversus second-side injection, epileptogenic versus nonepileptogenic side injection, and the relationship between dissipation of slowing and return to baseline. We examined our data for information about whether EEG or motor testing showed faster resolution of amobarbital effects. A regression analysis, using the interval between injections as a predictor of the electrographic recovery to baseline, was also performed.

\section{RESULTS}

The means and SDs for recovery of motor function and return to normal electrographic baseline are listed by category in Table 2. Overall, the mean latency to motor recovery was $6.4 \mathrm{~min}$, and the latency for return to electrographic baseline was $7.8 \mathrm{~min}$. In only 3 cases out of 73 with adequate documentation of motor recovery did motor recovery occur after electrographic recovery. Motor recovery was clearly longer after the second injection than after the first $(t=3.23, p<0.003)$. The side of injection did not have any influence on motor recovery time.

In terms of electrographic recovery, the interval to dissipation of the initial high amplitude delta activity did not correlate with any of the following variables: (a) the hemisphere injected, (b) the side of epileptogenesis, (c) the degree of baseline hemispheric slowing, or (d) the interval between injections. Frequencies over the hemisphere contralateral to the injection were usually normal before memory items were presented and always returned to baseline long before the injected hemisphere did. Examination of the latency to resumption of normal baseline frequencies in the injected hemisphere revealed

TABLE 2. Electrographic and motor recovery times $(s)$

\begin{tabular}{ll}
\hline & Mean + SD \\
\hline Electrographic & \\
Mean recovery to baseline EEG (all injections) & $465 \pm 121$ \\
Left hemisphere recovery & $475 \pm 132$ \\
Right hemisphere recovery & $457 \pm 113$ \\
First hemisphere recovery & $446 \pm 107$ \\
Second hemisphere recovery & $487 \pm 134$ \\
Epileptogenic hemisphere recovery & $445 \pm 126$ \\
Nonepileptogenic hemisphere recovery & $488 \pm 113$ \\
Motor & \\
Mean motor recovery (all injections) & $382 \pm 114$ \\
First hemisphere recovery & $357 \pm 102$ \\
Second hemisphere recovery & $408 \pm 121$ \\
Epileptogenic hemisphere recovery & $380 \pm 126$ \\
Nonepileptogenic hemisphere recovery & $387 \pm 113$ \\
\hline
\end{tabular}

SD, standard deviation. no significant difference in recovery times between the dominant and nondominant hemispheres. However, a significant difference was noted between recovery of the epileptogenic hemisphere and the nonepileptogenic hemisphere, with the slower recovery seen after injection of the nonepileptogenic hemisphere $(t=2.34, p<$ 0.024).

When the first and second injections were compared, a significant delay was observed in both motor and electrographic recovery times after the second injection. The duration of amobarbital-induced electrographic dysfunction after the second test correlated with the time between injections. The regression line suggested that recovery was equivalent if $44 \mathrm{~min}$ or more had elapsed between injections (Fig. 1). We looked selectively at interinjection intervals of above and below $40 \mathrm{~min}$, and were able to demonstrate that the delay in electrographic recovery after the second injection disappeared if the waiting time was $\geqslant 40 \mathrm{~min}$.

We found that time to recovery to electrographic baseline was longer after the second injection, and also after injection of the nonepileptogenic hemisphere (Table 3). To determine whether the order of injection or the laterality of the focus was the most important factor in recovery delay, we performed 2 further analyses. First, we found that the interval between injections for IAPs where the epileptogenic hemisphere was injected first was no different from that of the interval between injections when the nonepileptogenic hemisphere was injected first. Next, we determined that recovery to baseline after the second injection was significantly longer only when the epileptiform hemisphere was injected first. Thus, we concluded that electrographic recovery was most strongly delayed when the nonepileptogenic hemisphere was injected, and that this effect was abolished by allowing $40-45 \mathrm{~min}$ to pass between injections.

\section{DISCUSSION}

Electrographic and motor recovery have proved to be complementary tools during the IAP. Previous studies (10) have indicated an excellent correlation between quantitative EEG and the on-line visual analysis that provides helpful information during the procedure; our data and recent quantitative assessments at our center correlate well with Bouwer's findings.

On-line EEG analysis can indicate the degree and duration of ipsilateral and contralateral amobarbital effect and can clarify whether the drug has bilateral effects in unresponsive patients. It also elucidates unusual behavior and excludes ictal activity. No memory items are presented prior to the dissipation of high-amplitude, often diffuse, delta activity. At our center, motor function is used to gauge early recovery, and no memory items are presented after motor strength has returned to three-fifths 


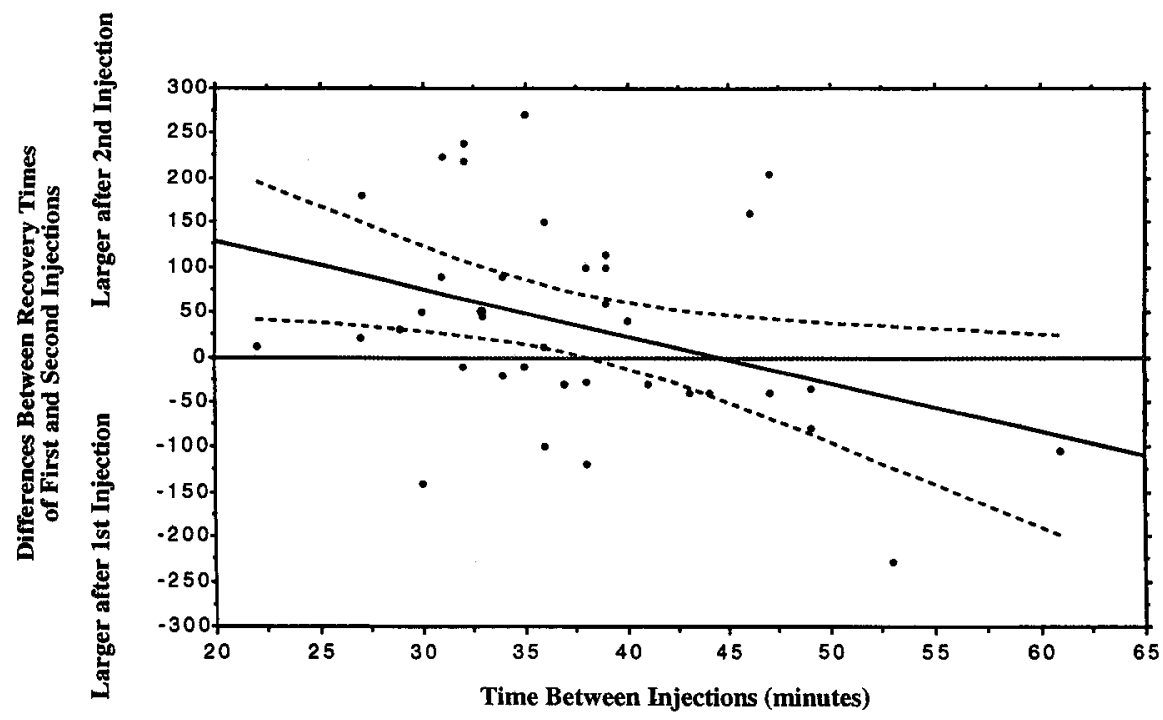

FIG. 1. Regression analysis of the relationship of time interval between injections and EEG recovery. Dashed lines are $95 \%$ confidence interval $(\mathrm{Cl})$ for regression line. or more. Testing for recall of items presented during amobarbital effect is undertaken only after the EEG has returned to baseline. With our protocol, motor function was recovered earlier than electrographic activity in 70 of 73 cases.

Resumption of normal baseline electrographic activity after amobarbital injection is an important event for a number of reasons. In this series, EEG proved to be a significantly more sensitive indicator of resolution of amobarbital-induced cerebral dysfunction than did motor recovery. Other authors have suggested that behavioral or cognitive abilities may return significantly later than motor and even language function (15), and our EEG data provide indirect support for this conclusion. The behavioral significance of electrographic recovery is evident in a study of short-term and long-term memory during IAP, which assessed recall of test items (a), while activities over the injected hemisphere were slowed, and (b), after electrocerebral function had returned to baseline (4). When subjects were tested before amobarbital effects had disappeared, memory scores did not clearly differentiate between epileptogenic and nonepileptogenic hemispheres, whereas testing after the EEG returned to baseline demonstrated a significant asymmetry in the memory function of the seizure-prone hemisphere. Testing prior to resumption of baseline EEG activity might therefore underestimate memory risk after surgery and provide less useful information for centers using the IAP to help confirm lateralization of the epileptogenic zone.

We observed a significantly longer latency to resumption of baseline electrographic activities following amobarbital perfusion of the nonepileptogenic hemisphere than after perfusion of the epileptogenic hemisphere. Similar findings were previously mentioned by Rausch and colleagues (16). These unexplained observations contradict a naive expectation that the more dysfunctional hemisphere would be slower to recover from an exogenous perturbation, such as supraphysiologic concentrations of a barbiturate receptor agonist. Given the widespread hemispheric amobarbital perfusion produced by intracarotid administration, the explanation of these observations might be approached in terms of mechanisms of recovery from amobarbital where they differ in the epileptogenic and nonepileptogenic hemispheres. Possible explanations of the briefer EEG and the motor effects of amobarbital on the epileptogenic hemisphere include: (a) a lower density of barbiturate receptors over critical regions of this hemisphere, (b) a lower affinity of barbiturate receptors for amobarbital over these regions, (c) a lower concentration of gamma-aminobutyric acid (GABA) over these regions (17), (d) other (unknown) mechanisms leading to slower reversal of late local effects of amobarbital in the nonepileptogenic hemisphere, and (e) other (unknown) mechanisms for reversal of late amobarbital effect mediated by the hemisphere contralateral to injection (i.e., resulting in a slower recovery when the more intact, nonepileptogenic, hemisphere is injected).

One can begin to distinguish between these potential explanations by assessing data from previous relevant studies. We have not seen reports of autoradiographic barbiturate ligand studies of resected tissue in partial epilepsies. However, the consistent colocalization of barbiturate receptors with GABA and central benzodiazepine receptors (cBZRs) in the $\mathrm{GABA}_{\mathrm{A}}$ receptorchloride ionophore complex (18) permits indirect assessment of barbiturate receptor concentrations in human tissue. Autoradiographic studies reveal consistent decreases in GABA receptor and CBZR density in the hippocampus but not in the neocortex of temporal lobectomy specimens (19-21). Further, positron emission 
TABLE 3. Recovery time comparisons (paired t tests)

\begin{tabular}{|c|c|c|}
\hline & $\mathrm{p}$ value & $\mathrm{t}$ \\
\hline \multicolumn{3}{|l|}{ Electrographic recovery } \\
\hline \multicolumn{3}{|l|}{ Hemisphere 1 vs. hemisphere 2} \\
\hline Right & NS & \\
\hline Second & $\mathrm{p}=0.027$ & 2.30 \\
\hline Epileptogenic Nonepileptogenic & $\mathrm{p}=0.024$ & 2.34 \\
\hline \multicolumn{3}{|l|}{ Interval between injections } \\
\hline Epileptogenic first vs. nonepileptogenic first & NS & \\
\hline \multicolumn{3}{|c|}{ Difference in recovery time (second minus first injection) } \\
\hline Epileptogenic first vs. nonepileptogenic first & $\mathrm{p}=0.024$ & 2.34 \\
\hline \multicolumn{3}{|l|}{ Motor recovery } \\
\hline \multicolumn{3}{|l|}{ Hemisphere 1 vs. hemisphere 2} \\
\hline First $\quad$ Second & $\mathrm{p}=0.003$ & 3.23 \\
\hline Epileptogenic Nonepileptogenic & NS & \\
\hline
\end{tabular}

NS, not significant.

tomography (PET) with the cBZR ligand $\left[{ }^{11} \mathrm{C}\right]$ flumazenil shows that cBZR density consistently is decreased in anterior mesial temporal regions and rarely decreased in temporal neocortex and extratemporal regions in limbic temporal lobe epilepsy $(22,23)$. It is difficult to explain widespread hemispheric asymmetries in amobarbital effect by highly focal hippocampal asymmetries in barbiturate receptor density or affinity. Previous studies of hippocampal recordings (24) have documented local amobarbital effects, and quantitative intracranial recordings might help to clarify whether local ligand-receptor interactions could affect the speed of recovery.

We have also explored the influence of successive IAP procedures on electrographic recovery time, and have found that the first injection can significantly delay the recovery to baseline EEG activities after a second injection within $20-40 \mathrm{~min}$. This would imply some type of residual effect of the injection. Given the previous studies by Rouleau (4) and Rausch (16), we feel it is possible that delayed electrographic recovery reflects slower cognitive recovery. It seems wisest to eliminate any potential behavioral effects of the first injection before testing the second hemisphere. Different centers have used a variety of techniques to improve same-day recovery, including the use of methohexital or titration of amobarbital doses. We have modified our practice to allow $45 \mathrm{~min}$ between injections. After $45 \mathrm{~min}$, we found no electrographic evidence that the first injection influenced recovery from the second injection. Our data support the possibility that both intracarotid amobarbital procedures might reasonably be performed with a single transfemoral catherization in one day.

\section{REFERENCES}

1. Wada J. A new method for determination of the side of cerebral speech dominance: A preliminary report on the intracarotid injection of sodium Anmtal in man. Iqaku to Seibutzuqaki 1949;14: $221-2$.
2. Milner B, Branch C, Rasmussen T. Study of short-term memory after intracarotid injection of sodium Amytal. Trans Am Neurol Assoc 1962;87:224-6.

3. Engel J, Jr, Rausch R, Lieb JP, Kuhl DE, Crandall PH: Correlation of criteria used for localizing epileptic foci in patients considered for surgical therapy of epilepsy. Ann Neurol 1981;9:215-24.

4. Rouleau I, Labrecque R, Saint-Hilaire J-M: Short-term and longterm memory deficit following intracarotid Amytal injection: Further support for the memory consolidation hypothesis. Brain Cogn 1989;11:167-85.

5. Rausch R, Babb TL, Engle J Jr, Crandall PH: Memory following intracarotid amobarbital injection contralateral to hippocampal damage. Arch Neurol 1989;46:783-88.

6. Rausch R, Langfitt JT: Memory evaluation during the intracarotid sodium amobarbital procedure. In: HO Luders (ed). Epilepsy Surgery. New York: Raven Press, 1992:507-15.

7. Perrine K, Westerveld M, Sass KJ, Devinsky O, Dogali M, Spencer DD, Luciano DJ, Nelson PK. Wada memory disparities predict seizure laterality and postoperative seizure control. Epilepsia 1995; 36(9):851-6.

8. Wyllie E, Naugle R, Awad I, Chelune G, Liiders Dinner D, Skibinski C, Ahl J: Intracarotid amobarbital procedure: I. Prediction of decreased modality-specific memory scores after temporal lobectomy. Epilepsia 1991;32(6):857-64.

9. Kneebone AC, Chelune GJ, Dinner DS, Naugle RI, Awad IA: Intracarotid amobarbital procedure as a predictor of materialspecific memory change after anterior temporal lobectomy. Epilepsia 1995;36(9):857-65.

10. Bouwer MS, Jones-Gotman M, Gotman J: Duration of sodium Amytal effect: Behavioral and EEG measures. Epilepsia 1993;34 (1):61-8.

11. Rausch R, Silfvenius H, Wieser H-G, Dodrill C, Meador KJ, Jones-Gotman M. Intraarterial Amobarbital Procedures. In: Engle Jr J (ed.) Surgical Treatment of the Epilepsies. New York: Raven Press, 1993.

12. Dinner DS: Intracarotid amobarbital test to define language lateralization. In: HO Luders (ed). Epilepsy Surgery. New York: Raven Press, 1992:503-6.

13. Henry TR, Ross DA. Summary of epilepsy surgery protocols: University of Michigan. In: Lüders H (ed.) Epilepsy Surgery New York: Raven Press, 1992:781.

14. Milner B. Psychological aspects of focal epilepsy and its neurological management. In: JK Penry \& RD Walters (eds.) Advances in Neurology (Vol 8). New York: Raven Press, 1975:299-321.

15. Aasly J, Silfvenius H: Evaluation of early and late presented tasks in the intracarotid Amytal test for epileptic patients. Epilepsy Res 1990;7:155-64.

16. Rausch R, Fedio P, Ary CM, Engel J Jr, Crandall PH: Resumption of behavior following intracarotid sodium amobarbital injection. Ann Neurol 1984;15:31-5.

17. During MJ, Spencer DD. Extracellular hippocampal glutamate and 
spontaneous seizure in the conscious human brain. Lancet 1993; $341: 1607-10$.

18. Savic I, Ingvar M, Stone-Elander S. Comparison of $\left[{ }^{11} \mathrm{C}\right]$ flumazenil and $\left[{ }^{18}\right.$ F]FDG ad PET markers of epileptic foci. J Neurol Neurosurg Psychiatry 1993;56:615-21.

19. Macdonald RL, Olsen RW. GABA receptor channels. Annu Rev Neurosci 1994;17:569-602.

20. Mcdonald JW, Garofalo EA, Hood T, et al. Altered excitatory and inhibitory amino acid receptor binding in hippocampus of patients with temporal lobe epilepsy. Ann Neurol 1991;29:529-41.

21. Johnson EW, de Lanerolle NC, Kim JH, et al. "Central" and "pe- ripheral" benzodiazepine receptors: Opposite changes in human epileptogenic tissue. Neurology 1992;42:811-5.

22. Burdette DE, Dakurai SY, Henry TR, et al. Temporal lobe central benzodiazepine binding in unilateral mesial temporal lobe epilepsy. Neurology 1995;45:934-41.

23. Henry TR, Frey KA, Sackellares JC, et al. In vivo cerebral metabolism and central benzodiazepine-receptor binding in temporal lobe epilepsy. Neurology 1993;43:1998-2006.

24. Gotman J, Brouwer MS, Jones-Gotman M. Intracranial EEG study of brain structures affected by internal carotid injection of amobarbital. Neurology 1992;42:2136-43. 